\title{
La religion dans le roman feuilleton allemand
}

The Author studies the mode of appearance of religion in the popular, serialized, German novel. Through the different versions of this literary genre (sentimental novel, medical novel, regional history, western...) the analysis permits the discovery of a functional, lived experience comparable to the religious experience habitually investigated by the social sciences with the aid of other indicators.

«Trivial religion» with this light shed upon it seems to correspond rather well to aesthetic or philosophical needs for security, the very simple structure of which accord with the role of the consumer as individual.

\section{LE PROBLEME ET LES OBJECTIFS}

Lorsqu'on pose la question de l'impact de la religion sur les sociétés hautement différenciées telles que la République Fédérale d'Allemagne (RFA), toute réponse sera bien sûr fort dépendante de ce que l'on entend par religion. Si on parle de phénomènes religieux chaque fois que l'on observe des mécanismes qui servent à faire face aux contingences de la vie, on ne parlera guère d'une perte de vitesse de la religion. Si on considère au contraire les phénomènes religieux comme des formes d'expressions humaines destinées à interpréter le monde et soi-même à l'aide des traditions des grandes religions mondiales, on sera plus facilement amené à constater une baisse d'importance de la religion par rapport à la place qu'elle tenait dans les sociétés préindustrielles. Si le concept de religion est lié étroitement aux formes d'organisation qu'ont prises les Eglises chrétiennes dans le monde occidental et nord-américain, on aura tendance à parler d'une importance actuelle plutôt faible de la religion - notamment si ce jugement est basé sur le critère de la participation aux rites prescrits par les Eglises. Cette problématique est largement connue. Toutefois, il ne semble pas encore exister de réponse satisfaisante à la question de l'impact réel de la religion dans un pays comme la RFA. Cela s'explique par des incertitudes non seulement sur le plan théorique des sciences humaines mais aussi sur le plan des méthodes empiriques de ces sciences. A travers ces méthodes, la religion est vue surtout comme attitude face à des questions religieuses. Normalement, le sujet interrogé ne s'exprime pas avec toute la spontanéité nécessaire sur le thème de la religion. Il le fait à travers ses réactions aux questions posées. Dans des méthodes employées plus récemment (et aussi 
plus rarement) au cours de recherches socio-religieuses - dans l'interview autobiographique, par exemple - la relation autobiographique ne semble pas non plus correspondre à la réalité quotidienne car elle demeure trop centrée encore sur une problématique préalablement orientée.

L'étude qui suit partira des expressions sociales de la vie courante et non des expressions produites au cours du processus communicatif d'une recherche méthodique. La question est la suivante: comment la religion se manifeste-t-elle dans les romans feuilletons allemands? Ces romans constituent, si l'on peut dire, une catégorie de lectures populaires dont l'importance est considérable en raison de leurs grands tirages; ils correspondent manifestement aux besoins et à la demande d'un grand nombre de lecteurs appartenant surtout aux couches sociales inférieures. On pourrait donc supposer que ces romans reflètent aussi la religion d'une grande partie de cette population. Aussi une analyse des romans devrait mettre en lumière des phénomènes religieux au-delà de ce qu'un sondage sur les attitudes religieuses peut découvrir. Pour englober toute la complexité des phénomènes religieux, notre concept de religion adoptera au départ la portée la plus vaste. Nous parlerons de religion chaque fois que se trouve symbolisé le problème de l'attitude face aux contingences de la vie et où l'on cherche à trouver ou à proposer des solutions à ce problème.

\section{LES ROMANS-FEUILLETONS ET LEUR DIFFUSION}

Les romans populaires sont proposés aux lecteurs sous formes de livres reliés, livres de poche ou brochures, la forme des publications étant souvent l'indicateur d'un niveau littéraire fort variable.

Publié sous forme de brochure, le roman-feuilleton comprend quelques 64 pages imprimées en deux colonnes; donc 120 pages de manuscrit environ. Actuellement, un tel roman se vend au prix de 1,70 DM. Certains romans s'adressent spécifiquement aux femmes: histoires de familles, destins d'enfants, romans d'amour, de "mon pays" (Heimat) ou de montagnes, de noblesse ou de médecins. Les romans du genre Western, policier, sciencefiction ou de guerre s'adressent plutôt aux lecteurs masculins. Depuis quelques années certaines séries visent surtout les jeunes femmes de par leur sujet et leur présentation (Julia, Romana, Bianca, Désirée etc., séries publiées en Livres de poche, cfr Speetzen, 1981).

Vers le milieu des années soixante-dix, le tirage hebdomadaire estimé s'élevait à 3,6 millions de romans pour hommes et 3,9 millions de romans pour femmes, le tirage total tournant autour de 370 millions par an pour la RFA (Speetzen, 1979). Selon des estimations, un tiers de la population allemande comptait parmi les lecteurs de romans-feuilletons, les taux étant plus élevés pour les femmes, les adolescents et les jeunes adultes jusqu'à 30 ans. La variation des taux selon la scolarité ne semble guère surprenante: en 1973, $15 \%$ des bacheliers (Abiturienten) se déclaraient lecteurs contre $34 \%$ chez les personnes de scolarité inférieure (Volksschule) (Schmidtchen, 1974, 773). Les séries les plus populaires sont les policiers, les romans d'amour et de "destin», des romans de médecins et les Western. 
Les romans sont écrits selon les directives des éditeurs. Actions et personnages doivent être présentés de façon stéréotypée. On favorise un certain «réalisme»; une attitude critique face à la société est peu souhaitée. Dans les directives d'une commission d'auto-contrôle créée par les éditeurs on peut lire: «Administrations, pouvoirs publics et personnages qui représentent le droit et la morale (juges, prêtres, agents de police etc.) sont à présenter de sorte que l'on puisse les identifier comme groupes ou personnages positifs» (Pforte, 52).

En général, ces romans ne sont pas vendus en librairie mais dans les kiosques, papeteries et supermarchés. En dépit du grand nombre de séries, ce sont surtout deux éditeurs qui dominent le marché allemand (Bastei, Bergisch Gladbach et Heinrich Bauer, Hamburg, cfr Pforte, 34).

Cette distribution particulière des romans-feuilletons s'inscrit dans la tradition des lectures populaires en Allemagne (littérature de colportage, Traktatgesellschaften, voir Schäfer et surtout Schenda). Dans son genre littéraire également, le roman populaire d'aujourd'hui est bien enraciné historiquement au 19e siècle. Karl May (1842-1912), par exemple, avait publié des romans du genre Western avec grand succès, le roman pour femmes étant lié étroitement aux noms d'Eugenie Marlitt (1825-1887) et Hedwig Courths-Mahler (1967-1950, auteur particulièrement productive). Dans le domaine du roman se jouant en milieu alpin le nom de Ludwig Ganghofer (1855-1920) est célèbre. On pourrait aisément prolonger cette liste d'auteurs toujours lus ou oubliés depuis.

Du côté de la RDA on observe également l'existence d'une littérature du roman trivial, bien que dans des quantités plus limitées. Toutefois, on estime à quelques 500.000 exemplaires par mois le nombre de romans vendus dans les kiosques. En RDA, les romans s'adressent principalement aux adolescents et aux hommes et, curieusement, on constate l'absence de la littérature du genre romans d'amour et de mariage, genre destiné aux femmes (Mallinckrodt, 10 et 13 ).

\section{PROJET D'ETUDE}

Depuis la fin des années soixante la littérature triviale a fait l'objet d'une attention accrue. Ceci est sans doute imputable au changement de perspective dans les sciences littéraires qui passèrent d'une analyse trop exclusivement esthétique (cfr Killy) à une perspective plutôt socio-historique. En plus, la littérature triviale paraissait intéressante en tant que littérature non d'une élite mais des masses (Kaes/Zimmermann et Kreuzer). De ce fait, on commença à étudier aussi les romans-feuilletons en tant que «romans destinés aux classes inférieures» (Nusser). Vu sous l'aspect de l'analyse fonctionnelle, c'est l'approche de la critique idéologique qui s'est avérée fertile: en raison des conditions de vie aliénantes, ces romans apparaissent comme moyens de compensation des frustrations subies (cfr Bürger et bien d'autres). On conçoit donc l'existence d'un certain parallélisme avec les fonctions de la religion. Ce qui a fait soulever dans un bon nombre d'études la question plus particulière des implications religieuses dans ces textes. Or, ces études sont moins centrées sur le roman populaire que sur d'autres catégories littéraires. Ainsi, Heinrich 
Krauss traite le thème religieux dans la science-fiction, Wermke la dimension religieuse dans les bandes dessinées (voir Wermke, Ohlig, Groeben). Des questions analogues ont été étudiées dans l'analyse de films fantastiques (Giesen). En ce qui concerne les romans-feuilletons en Allemagne, l'étude de Marianne Jabs-Kriegsmann sur le roman des revues illustrées des années 1950 à 1977 (voir aussi l'étude de Metz dans une perspective d'église) semble intéressante car elle consacre un chapitre entier au thème de la religion. Anita M. Mallinckrodt ne traite pas le sujet dans son étude des romans-feuilletons en RDA car, dans une perspective socialiste, les romans en RDA passent sous silence la religion sans même, comme on aurait pu le penser, la traiter comme adversaire.

Du point de vue de la sociologie des religions, ces études amènent à poser deux problèmes importants : d'une part, l'identification des énoncés touchant à la religion et à l'Eglise par la méthode d'analyse du contenu; d'autre part et de manière plus importante, l'analyse fonctionnelle de cette production littéraire.

S'il est vrai que les romans-feuilletons peuvent assumer des fonctions compensatrices, leur lecture ne serait-elle pas l'équivalent fonctionnel d'une consolation religieuse qui, elle aussi, est souvent qualifiée par les critiques de la religion comme une solution compensatrice et illusoire des problèmes qui sont à résoudre par l'action politique? Nous avons essayé de tenir compte de ces deux problèmes dans la courte analyse suivante.

Nous avons examiné 118 romans, en vente dans les kiosques entre octobre 1980 et janvier 1981. Il ne s'agissait pas d'un échantillonnage systématique, mais nous avons tenu compte dans notre sélection de l'importance des différents genres de romans sur le marché (romans de familles, de noblesse, policiers etc). L'analyse de contenu a été faite selon un inventaire standard. Les principaux personnages ont été évalués à l'aide de profils de polarités. En plus, des procédés d'échelles (Likert et Guttmann) ont été utilisés.

L'analyse du contenu portait sur les phénomènes religieux explicitement nommés dans les textes (y compris les éléments non-chrétiens: or, des études préliminaires avaient déjà montré l'appartenance ou la proximité de toute allusion explicite - ou presque - au christianisme). Ensuite, nous avons essayé d'évaluer le caractère général de chaque roman selon les critères suivants : problèmes posés au cours de l'action et solutions proposées; qualité des personnages; présentation éventuelle d'un monde "beau et gentil»; souci éventuel d'assoir la légitimité des institutions sociales et l'ordre des valeurs dominantes.

Trois ans après (printemps 1984), nous avons examiné à l'aide du même inventaire 19 romans plus anciens, réédités et actuellement en vente sous forme de livre de poche. Il s'agissait, à quelques exceptions près, de romans des auteurs Karl May, Eugenie Marlitt et Hedwig Courths-Mahler, pour la plupart écrits entre 1880 et 1920.

Selon les affirmations des éditeurs (Lübbe, Pawlak, Deutscher Literaturverlag) les textes n'ont pratiquement pas été abrégés. Par la comparaison avec les romans actuels et plus anciens - mais toujours lus -, cette étude supplémentaire voulait faire ressortir les changements intervenus en ce qui concerne la place de la religion. 
Dans certains cas, des analyses factorielles ont été exécutées (Pastoralsoziologische Arbeitsstelle Hanovre, W. Lukatis). Des sommaires des actions de romans ont été établis ainsi que des registres des matières religieuses. A côté de l'analyse de contenu, des discussions ont eu lieu entre les personnes chargées d'établir les inventaires (étudiants en théologie protestante à l'université de Göttingen) pour étudier les réactions subjectives auprès du lecteur.

Nous ne présenterons que très sommairement les résultats de l'analyse de contenu standardisée, mettant plus d'accent sur l'analyse des schèmes d'actions, typiques des romans-feuilletons.

\section{RELIGION EXPLICITE DANS LES ROMANS-FEUILLETONS}

Dans presque les trois quarts des romans examinés on trouve au moins des vestiges d'un langage religieux, vestiges englobant ici des éléments éphémères tels que les symboles religieux mentionnés dans des exclamations (Mon Dieu! Ciel! etc.). On est surpris de constater que presque un tiers des romans se réfère plus ou moins explicitement à des symboles religieux pour interpréter des expériences de la vie. Si l'on tient compte aussi des symboles non-chrétiens explicitement nommés et du langage "post-chrétien " (ainsi que, par exemple, dans le terme "destin») on arrive à un taux de $43 \%$ des romans étudiés qui proposent de telles interprétations. C'est la prière - l'oraison jaculatoire surtout - qui joue le rôle le plus important parmi les différentes formes de la pratique religieuse chrétienne. Sous une forme ou sous une autre, la prière intervient dans un tiers de tous les romans.

D'autres formes de la pratique chrétienne par contre, la participation au culte par exemple ou les allusions aux fêtes liturgiques, se rencontrent bien plus rarement. Bien qu'un cinquième environ des romans aient été publiés en décembre 1980, la fête de Noël n'est mentionnée que dans huit cas.

Des éléments du contenu de la tradition chrétienne - le Décalogue ou des récits du Nouveau Testament, par exemple - sont mentionnés dans $21 \%$ des romans. Dans $14 \%$ des cas, un rapport est établi entre la conduite morale des personnages et les normes ou modèles religieux. $6 \%$ des romans seulement parlent de traditions religieuses non chrétiennes.

Dans quatre romans seulement le personnage sauveur de Jésus Christ est nommé explicitement. Ceci ne permet pourtant pas de conclure que la religion des romans-feuilletons - quoique formulée dans un contexte chrétien - est une religion sans le Christ. Toutefois, on a l'impression que le personnage du fondateur ne joue pas un rôle éminent. En parlant de religion - si l'on en parle - on se réfère plutôt à un contexte plus généralement chrétien.

On rencontre, bien sûr, des personnages de profession religieuse: le pasteur protestant dans un quart des romans, le prêtre catholique dans $30 \%$ des cas, les religieuses dans des cas plus rares. Dans deux tiers de ces cas, l'importance générale de ces personnages pour le déroulement de l'action est jugée plutôt faible alors que dans $20 \%$ des cas ils prennent une fonction assez importante. D'autres professions acquièrent une fenction quasi religieuse: le médecin notamment, l'infirmière plus rarement.

En s'interrogeant sur la signification des institutions sociales, on constate qu'il n'est guère question de l'Eglise. Le mariage et la famille, l'ordre du droit et de la propriété représentent les institutions affirmées par priorité. Résultat 
assez analogue en ce qui concerne les valeurs dominantes: La «foi religieuse» y occupe le dernier rang d'une échelle dont les premiers appartiennent à l'entraide, la générosité, l'accomplissement du devoir, le courage et l'honnêteté. Ici une comparaison des romans actuels avec ceux du passé paraît significative; pour deux valeurs on observe un changement de rang sur l'échelle: dans les anciens romans la foi religieuse et la «fidélité du mariage» occupent une place plus importante. Ce rôle relativement plus important de la foi religieuse dans les anciens romans se confirme par la plupart des autres indicateurs qui donnent une place relativement plus importante au thème de la religion.

De plus, les analyses qualitatives de contenu mettent en relief que, dans les anciens romans, la religion (au sens des Eglises chrétiennes) fait partie des réalités sociales évidentes, réalités avec lesquelles on peut s'identifier. Les romans d'Eugenie Marlitt, par exemple, laissent entrevoir que l'auteur se trouve aux prises avec les différents courants à l'intérieur de son Eglise (protestante) puisqu'elle s'élève contre un certain piétisme. Des positions aussi différenciées ne se trouvent pas - ou plus - dans les romans actuels.

Ce sont surtout les romans pour femmes qui abordent le thème de la religion. Prise au sens de la religion traditionnelle chrétienne, elle y joue un rôle bien plus important que dans les romans du type masculin.

Prenons deux exemples de romans pour illustrer comment le thème religieux y est introduit par l'intermédiaire de personnages de prêtres. Le roman "C'est pour toi que j'ai dit oui » (série "Mutterglück», Marken-Verlag) nous raconte l'histoire de Silke Brahmkamp, fille de pasteur protestant. Elle est employée dans une grande ville en Allemagne du nord, mais elle habite toujours la maison paternelle. Un jour, elle fait connaissance de son futur patron qui sera aussi son mari. Le soir à la maison se déroule l'épisode suivant:

"Toute la famille était déjà réunie autour de la table dans la salle à manger de la maison d'Olversen lorsque Silke rentra. Dans cette famille de cinq enfants on mangeait toujours à l'heure, le soir. De temps en temps, au cours du repas, le pasteur Gerhard Brahmkamp regardait sa fille avec attention. Elle lui paraissait bien silencieuse et songeuse ce soir.

- Tu as passé une lourde journée, Silke? lui dit-il, préoccupé.

La jeune fille regarda son père d'un air étonné.

- Non, pas du tout. Mais aujourd'hui, mon futur patron est venu se présen-

ter et il a donc obtenu ce poste. Il va commencer dès la semaine prochaine.

- Il a l'air sympathique?

- Oh oui, on peut le dire.

- Il est jeune ou déjà plus âgé?

- Très jeune, il a 32 ans seulement. Il semble très ambitieux.

- As-tu peur de ne pas être à la hauteur de ses exigences?

- Pas vraiment, papa, mais il faudra voir. Ce n'est quand même pas simple de s'habituer à quelqu'un d'inconnu.

- Je te comprends, Silke, mais tu le verras: tout se passera bien. Quand on est toujours bien ponctuel et appliqué à son travail on obtient de l'estime et de la reconnaissance chez tout le monde, dit le pasteur pour la rassurer.

Silke était aussi de cet avis.

- Alors, dans notre prière commune, nous demanderons ce soir que le Seigneur te donne son secours, dit le pasteur. Il semble que tu en auras besoin. Il plia les mains pour la prière et toute la famille fit comme lui. Ils étaient tous très attentifs et le silence fut absolu pendant la prière du père. 
C'était ainsi tous les jours. Silke ne l'avait jamais connu autrement. Les mouvements et les modes passaient à côté de cette maison sans jamais rien y changer. C'était toujours le même style de vie dans la maison du pasteur et cela avait quelque chose d'infiniment rassurant ». (p.8)

On apprend plusieurs choses sur la maison du pasteur protestant : les éléments y sont assez stéréotypés. Le pasteur a de nombreux enfants; c'est l'ordre qui règne dans sa maison; la prière se dit en commun; les modes passent à côté. Voilà l'image qui représente un comportement conforme au sens de l'Eglise chrétienne.

Le second exemple est tiré d'un roman de noblesse ( «L'amour — pour moi cela n'existe pas ", Marken-Verlag). Un été de grande sécheresse, le comte Norbert von Kerina refuse aux paysans l'accès à la rivière. Le prieur d'une abbaye voisine du château intervient en faveur des paysans. Il se rend au château où le compte le reçoit avec plaisir:

"Que Dieu vous bénisse, Monsieur le Comte, dit le Père Aurelius simplement. - Venons aux choses sérieuses, exige Norbert en se tournant vers le moine. Il le regarde d'un air froid. Les rayons du soleil entrent par la fenêtre, traversent la pièce et mettent en relief les traits profonds du visage du Père Aurelius, dont la peau, tendue sur les joues, ressemble à du vieux parchemin. Ses yeux restent à l'ombre des sourcils touffus.

- Vous savez bien pourquoi je suis venu, j'en suis sûr, dit le prieur.

Norbert fut surpris de la voix sonore de cet homme si âgé.

- Ce n'est pas pour l'abbaye que je viens vous demander une faveur, dit le Père Aurelius. C'est l'existence même des paysans qui est en cause. Jamais depuis des siècles on n'a connu un été aussi sec. Les paysans ont peur de perdre les récoltes. Vous disposez de l'eau en quantité suffisante, Monsieur le Comte, car la rivière vient alimenter le lac. Il suffirait d'en dévier une partie et tout le monde serait sauvé.

- Et ce serait donc à moi de rester au sec, n'est-ce pas? Norbert fait un geste furieux. Oh non, je ne suis pas d'accord! Je n'ai pas seulement des terres, j'ai aussi une écurie, mon Père: plus de 50 chevaux à abreuver tous les jours.

- Aidez les paysans, supplie le prieur, Dieu vous récompensera.

- Si Dieu le voulait, il ferait tomber de la pluie, réplique Norbert von Kerina sur un ton cynique. Pourquoi ne vous adressez-vous pas à votre Dieu au lieu de venir chez moi, le Comte de Kerina?

Norbert von Kerina, resté intangible devant l'appel ardent du moine, appuie sur une sonnette. Franz, son valet apparaît et Norbert lui dit froidement :

- Le prieur désire partir. Accompagnez-le, s'il-vous-plaît.

- Vous vous rendez coupable d'un péché, Comte Kerina, dit le Père Aurelius. Car vous savez ce qui est écrit dans la Bible: Ce que vous aurez fait au plus humble de mes frères, c'est à moi que vous l'aurez fait. Peut-être allez-vous encore repenser tout cela.

D'un sentiment de découragement, le Père Aurelius quitta le château de Kerina.

Dans cette scène encore on retrouve les traits typiques d'une image positive du prêtre: c'est ici le moine, l'homme spirituel et ascétique qui se trouve face au comte impitoyable en intercesseur pour les paysans, proclamant l'évangile et lançant un avertissement prophétique.

Ni le pasteur protestant ni le moine catholique ne sont présentés comme personnages antipathiques - bien au contraire. Les représentants de l'Eglise se montrent comme les gardiens de l'ordre et de la justice. Les romansfeuilletons, nous l'avions dit, ne veulent nullement critiquer les Eglises. 
Lorsque le domaine de la foi chrétienne joue un rôle dans de tels romans, c'est donc souvent à travers des représentants des Eglises chrétiennes - tels que le pasteur Brahmkamp et le Père Aurelius - qu'il est introduit. Remarquons aussi que c'est surtout dans les romans du genre «Mon pays» que l'on rencontre des allusions au monde chrétien. L'Eglise y occupe sa place, bien ancrée dans la vie quotidienne en montagne. En résumant ces deux aspects, on peut donc constater que les auteurs de romans placent le sujet religieux (au sens chrétien) dans un monde à part, soit dans le monde des représentants ecclésiastiques, soit dans des pays lointains par rapport au monde moderne, dans un pays où les anciennes traditions sont toujours respectées.

Dans son étude des romans de revues illustrées des années 1950 à 1977, Jabs-Kriegsmann en arrive à constater que «dans ces romans bien des prêtres prennent les rôles de confesseurs ou de personnages apportant leur aide dans des situations de détresse. Plus ils s'engagent en faveur des autres, plus ils apparaissent «bons»; plus ils semblent détachés de la vie quotidienne de leurs Eglises, plus ils impressionnent» (p. 241). Dans notre deuxième exemple, le prieur correspond assez bien à cette image. La tendance décrite par JabsKriegsmann se confirme, en effet, dans les romans-feuilletons, notamment dans les romans récents. Jabs-Kriegsmann note un changement remarquable dans l'image du prêtre des romans d'illustrés depuis 1950, à savoir une chute de sympathie pour presque tous les personnages spirituels. A partir de 1965, on rencontre plus souvent des représentants d'Eglises peu sympathiques ou même ridicules. Or, à cette période, les ruptures des années '68 s'annoncent déjà assez clairement. En revanche, il paraît possible que, entre temps, le prêtre sympathique ait retrouvé sa place dans les romans de revues illustrées.

Dans l'ensemble, on peut donc dire que le monde de la religion - dans sa composante ecclésiastique et chrétienne surtout - occupe assez nettement sa place dans les romans, l'institution de l'Eglise étant symbolisée surtout à travers ses représentants. On constate avec surprise que, parmi les formes d'expression de la pratique religieuse, c'est la prière - l'oraison jaculatoire en situation d'extrême urgence notamment - qui tient la place prépondérante. Plus un élément a un caractère spécifiquement chrétien moins il apparaîtra dans les romans : ce qui est vrai aussi en ce qui concerne l'idée de Dieu. Souvent les romans préfèrent employer des termes de «destin » ou de "ciel» comme symboles d'une puissance supérieure qui détermine la vie. Ainsi, le concept de Dieu perd de son contenu spécifiquement chrétien alors qu'il est maintenu dans un sens plus vaste comme formule interprétative.

\section{RELIGION ET ACTION DE ROMAN}

Le rapport explicite aux traditions religieuses n'est qu'un aspect de notre problème. Ce qui est plus important ce sont les structures dans l'action des romans qui, sans lien manifeste avec le thème de la religion, font sousentendre une certaine "proximité» à la religion qui demande à être précisée davantage. Qu'est-ce qui fait penser à une telle proximité?

La dramaturgie des romans est souvent dominée par la mise en opposition de deux personnages qui représentent le bien et le mal. C'est le cas pour $62 \%$ de nos romans. Le drame se joue donc en priorité en raison de la tension 
entre le bien et le mal : c'est le bien qui s'impose, c'est le mal qui est vaincu. Le monde redevient un «monde salutaire» dès que l'adversaire malin est éliminé $(57 \%)$, dès que la personne en péril est libérée et sauvće $(57 \%)$. Il existe, bien sûr, d'autres issues dans les romans: la solitude surmontée, le partenaire trouvé pour la vie, la réussite et le bonheur dans la famille et dans le couple. Toutefois, ces issues également - typiques surtout des romans pour femmes - s'accordent implicitement au schème fondamental de cette opposition entre le bien et le mal qui finit par la victoire du bien sur le mal, par l'avènement du bonheur et du salut.

Prenons deux exemples pour illustrer ce thème éminent de la libération:

Dans le roman «Course contre la mort» (Bastei-Roman «Lassiter » (vol. 119), Lassiter est accusé d'avoir assassiné un colonel de l'armée. Cette accusation a été provoquée par Concho, ancien chef indien et ennemi de Lassiter. Concho veut venger son fils, un assassin que Lassiter avait livré à la justice. Concho lui-même a tendu le piège au colonel de sorte que Lassiter soit soupçonné coupable d'un crime. Le colonel est tué dans l'embuscade et sa fille est enlevée. Lassiter promet au colonel mourant de libérer sa fille. Or, des obstacles presque insurmontables se dressent devant lui. Il s'allie à un chasseur d'hommes, mais celui-ci cède bientôt aux charmes de la belle fille de Concho. Après bien des aventures, Lassiter atteint son but. Il peut alors rendre à son propriétaire la fortune dont l'ennemi s'était emparé de manière criminelle. La fille du colonel est libérée. La fille de Concho, pour sa part, se révolte contre son père et regagne le chemin de la justice. Lassiter est le héros qui doit souffir injustement, qui doit traverser des difficultés mais qui est finalement le vainqueur, et de ce fait capable de libérer les autres.

Le second exemple est tiré du roman de médecin (Marken-Arztroman, vol. 796) titré: “La patiente est-elle vraiment malade?» Le riche William Schönfeld fait connaissance du Docteur Wernicke, médecin dans une clinique neurologique. A l'occasion d'une invitation chez Schönfeld, Wernicke rencontre Viola Schönfeld, fille de William. Jugée comme épileptique, cette fille vit quasiment prisonnière dans la maison de son père qui, lui, est remarié. Viola soupçonne que son père et sa seconde femme essayent de lui soustraire l'héritage de sa mère. Wernicke lui propose son aide. L'aide sera très vite utile, car Viola se voit hospitalisée dans la clinique du Docteur Marius, médecin de réputation sinistre. Encouragé par la nourrice de Viola, Wernicke prend congé de son travail pour demander un poste - justement libre - dans la clinique de Marius. La clinique se révèle un véritable lieu de torture pour les patients. A des prix exorbitants, Marius fournit des expertises qui servent à déshériter des patients. Wernicke réussit à enlever Viola de la clinique. Son traitement ultérieur prouve que les crises d'épilepsie n'étaient que de caracètre léger et passager. L'histoire peut donc se terminer par le mariage heureux de Viola et Wernicke.

Ces deux romans apparemment, si différents, révèlent une structure commune:

1. ils se déroulent sur un arrière-fond dualiste: d'un côté les bons, de l'autre côté les méchants; de part et d'autre les représentants des puissances qui s'affrontent.

2. la lutte entre le bien et le mal fournit le sujet principal. Dans les deux cas, la solution se trouve dans la victoire du bien. 
3. la lutte entre le bien et le mal met en cause des personnes prisonnières innocentes qui sont les véritables victimes. La victoire se réalise par la libération de ces victimes. La victime innocente ne souffrira plus.

4. Dans la lutte entre le bien et le mal, le bien est représenté par le libérateur qui, lui, met sa propre vie en jeu. En se sacrifiant il peut donc se retrouver lui-même chargé du rôle de la victime innocente.

Ainsi, sans le moindre emploi d'un langage religieux, la vie humaine se trouve comme condensée à l'instar des contes de fées ou des systèmes interprétatifs religieux et dualistes: l'homme s'y trouve exposé aux conflits des puissances, dont l'une est bonne et l'autre est maligne. L'une le tient prisonnier, l'autre le libère: le salut se réalise.

Cette dramaturgie semble justement être la source de la fascination qu'exercent les romans sur le lecteur. Dans une tentative de m'observer moimême au cours de la lecture de certains romans, j'ai ressenti des difficultés à faire une pause de lecture lorsque l'auteur avait construit une action de suspense. Quand tout était bien fini, quand le héros avait remporté la victoire, la victime était libérée, les amoureux s'étaient retrouvés, je pouvais enfin poser mon roman, soulagé. Nos étudiants à Göttingen chargés de lectures et de codages ont parlé d'expériences semblables disant, par exemple:

- J'ai souffert, j'ai espéré avec le héros dans sa détresse. J'ai ressenti de la compassion et du soulagement au moment du dénouement. J'étais bien content que le méchant soit puni.

- Pour moi, c'était important qu'il y ait, à la fin, des solutions simples. Pour une fois, les choses compliquées ne se compliquaient pas davantage.

- C'est après la lecture seulement que j'ai réalisé que j'étais là pour analyser. D'abord je voulais arriver à la solution qui était formidable.

- Ça m'a contrarié d'être autant fascinée par l'histoire. Je me suis laissée prendre dans mon propre désir d'un monde gentil. Au moment de l'analyse seulement, $\mathrm{j}$ 'ai réussi à reprendre de la distance.

Il y avait aussi des réactions plus distancées comme celle-ci: J'ai trouvé impossible de m'identifier à ces personnages. Ce monde construit me paraissait trop artificiel - sauf peut-être dans les romans de montagnes. Je crois que parfois je rêve moi-même d'être Robinson Crusoë ou de vivre dans une vallée lointaine.

Ces observations semblent d'autant plus remarquables qu'elles sont exprimées par des étudiants - souvent saisis par un intellectualisme exagéré - qui, en plus, avaient lu ces romans dans une intention analytique. Or, même ces étudiants se montraient fascinés par un monde simple et transparent où l'homme honnête, juste et modeste, s'il doit souffrir, ne le fait que pour vaincre en définitive. Manifestement, la dramaturgie des romans correspond bien aux désirs secrets de beaucoup de personnes, au désir du simple, du sain, d'une fin de toute injustice, d'une consolation, au désir d'avoir droit aux larmes et de les savoir essuyées. La dramaturgie du roman déclenche une dramaturgie auprès du lecteur qui, de son côté, passera par des phases de peur, de tristesse, de compassion, et qui par ce cheminement passera d'un monde de la peur à la «terre promise» de la consolation, du bonheur, de la réalisation de ses voeux secrets. Des puissances malignes s'opposeront à lui, mais il sera soutenu par les bonnes puissances : le médecin compréhensif, le héros qui lutte pour la justice et qui souffre injustement. 
Dans des recherches sur les mass media on a posé la question des «gratifications" ressenties par les auditeurs et spectateurs des émissions de radio et de télévision. Selon Schenk, qui cite des résultats d'études au sujet des «soap operas » en Amérique, les auditrices de ces émissions journalières attendent les "gratifications» suivantes:

- une compensation substitutive de la non-réalisation de leurs propres voeux et rêves,

- une compensation de leur propre situation par identification avec des styles de vie plus profitables,

- la possibilité de projeter ses propres échecs sur les personnages de l'action, - de bons conseils en vue d'une meilleure réalisation des propres rôles (p. 218).

Des recherches sur la communication (Maletzke, 1525s) ont révélé que les processus d'identification et de projection se produisent au cours même de la perception des drames narrés. L'organisation dualiste des matières dramatiques ouvre en grand les voies d'accès à des possibilités d'identification et de projection et en accentue l'effet.

Certaines études ont signalé des parallélismes frappants avec la théorie du drame chez Aristote (voir Lipp, 370ss - nous faisons abstraction ici des questions théoriques posées par Lipp). L'effet du drame est décrit, chez Aristote, par le terme "katharsis». Le spectateur s'y trouve "purifié», soulagé, remis dans un état d'équilibre qui stabilisera son futur comportement. La katharsis se fait par une identification mais aussi par la prise de distance, dans l'angoisse, face au héros. "Le héros agit de façon pathique-pathétique sur 'l'exceptionnel' et sur la tension dont le groupe entier cherche à éviter le danger frémissant; il agit à la place des autres en chargeant sur ses propres épaules le poids du destin qui, en vérité, pèse sur tout le monde. Sa souffrance devient ainsi acte libérateur, acte 'purificateur'. Le groupe dont le héros a quitté les rangs observe cette action; par son sacrifice le héros non seulement préserve le groupe du mal, mais il le sauve pour un niveau d'existence plus élevé, pour une réalité de plus haut degré culturel qui donne accès à de nouveaux objectifs, de nouveaux contenus, de nouvelles libertés » (Lipp, 373). Participer à ce processus dramatique, c'est provoquer la katharsis et donc un soulagement. Notons en plus que le drame antique est une action conforme à l'ordre établi et garanti par les dieux qui en même temps est contesté par le héros. C'est en tout cas une forme d'expression d'une assurance religieuse ce qui peut se dire aussi de l'expérience vécue du spectateur. En ce sens, le drame est à la fois mythe présenté et mythe vécu, mythe spécifiquement religieux. Dans la dramaturgie des romans-feuilletons, la structure religieuse est moins manifeste. Mais, compte tenu de la structure de l'action et des réactions auprès du lecteur, ne serait-il pas légitime de considérer ces romans comme des histoires dramatiques chargées d'une symbolique qui serait en mesure de proposer aux lecteurs des orientations et des assurances? Ne faudrait-il pas y appliquer des concepts qui relèvent de la théorie des religions? Ces romans ne racontent-ils pas de nouveaux mythes - ou bien les anciens mythes sous une nouvelle forme? Ne seraient-ils pas l'expression de ces mythes du quotidien que Barthes a tenté de décrire et d'analyser? 


\section{HYPOTHESE HEURISTIQUE: LA REALITE NAREE COMME EXPRESSION DE RELIGION}

Au cours de notre analyse une idée s'est précisée de plus en plus clairement: non seulement il faut parler de la religion dans les romans-feuilletons mais aussi de la religion des romans-feuilletons. Mais faisons d'abord un pas de plus dans nos réflexions. Supposons de façon heuristique une présence implicite de la religion dans la plupart des romans-feuilletons, notamment lorsqu'ils sont construits selon une dramaturgie dualiste. Nous examinerons cette hypothèse en nous interrogeant d'abord sur les analogies structurelles entre la religion explicite et la "religion du roman-feuilleton» hypothétique. Dans une seconde démarche, il faudra examiner les différences de structure.

Une première observation concernant les analogies structurelles se réfère à une particularité du langage des romans: c'est un langage narratif qui, en même temps, va au-delà de la réalité narrée. Lassiter n'est pas que Lassiter: c'est le symbole même du héros juste. Dans le roman de médecin, c'est le personnage du médecin, dans les romans du genre «mon pays» ou de sciencefiction c'est tel ou tel autre personnage qui prendra ce rôle de héros. Puisque le lecteur retrouve constamment cette même structure du héros, garant de la justice, le personnage en question devient pratiquement transparent du héros comme tel. En reprenant les traditions plus anciennes on peut donc parler ici d'un langage symbolique ou mythique malgré les divergences actuelles dans les définitions de ces termes (cfr Barthes, 85ss; voir aussi l'emploi du terme de symbole chez l'Américain Gerbner dans les recherches sur les mass media: Albrecht, 96ss). Si par symbole on entend un signe qui renvoie au sens ultime d'une réalité, tout en parlant d'une réalité contingente et perceptible, et si par mythe on entend la narration d'actions contingentes qui renvoient à des réalités ultimes, on peut parler de langage mythique dans la plupart des romansfeuilletons. Le héros y représente une puissance qui garantit la justice ultime; s'il est en danger, c'est cette justice même qui est menacée; sa victoire vient exprimer la supériorité ultime de l'ordre de la justice. Le langage classique de la religion est un langage symbolique. Le narrateur de récits religieux parle sous forme de paraboles et de mythes. Aussi fictif que soit le langage symbolique des romans, il participe à un domaine linguistique qui s'efforce d'exprimer la structure profonde de la réalité.

La seconde analogie structurelle avec les phénomènes religieux est la suivante: souvent le roman-feuilleton suppose l'existence d'une puissance ultime qui détermine la vie appelée dieu, destin, ciel etc. Ce n'est pas par hasard que les «romans de destin » en viennent à constituer un genre ou une série particulière. Ces romans expriment l'expérience d'une puissance supérieure qui intervient dans la vie humaine: elle échappe à l'homme qui lui est exposé; elle lui inflige le malheur ou le fait vivre un bonheur merveilleux. Le dualisme du bien et du mal entraîne souvent une symbolisation dualiste de cette puissance dans le sens presque gnostique d'un affrontement entre Dieu et le Malin. Fait intéressant, au moment où le diable perd son rôle dans la prédication ecclésiale, il réapparaît sous les formes les plus variées dans les romans-feuilletons. En effet, des séries entières de romans se basent sur la présentation de cette puissance maligne: les romans d'épouvante, de fantômes, de science-fiction. 
C'est dans les romans de montagnes que nous trouverons une autre version du symbolisme de cette puissance supérieure expérimentée ou supposée: la montagne elle-même devient symbole de cette supériorité. En ceci, le roman actuel se trouve dans le sillon des traditions plus anciennes de la littérature triviale («Der weiße Tod - La mort blanche» de Rudolf Stratz, pour nommer un exemple qui a connu de nombreux tirages).

Une troisième analogie structurelle se situe dans la présentation du phénomène de la rédemption. Les romans débordent d'espoir de rédemption! Le sujet de la «Heimat », par exemple, implique tout un monde d'ordre, de sécurité, de respect, un monde limité, familier et transparent. De nombreuses séries racontent des histoires d'amour heureux: amour entre parents et enfants (le bonheur maternel), amour du couple qui partage le bonheur. Dans une société qui semble tant menacer la réussite des relations amoureuses, l'espoir d'une perpétuité de l'amour s'accentue de plus en plus. Un autre espoir de rédemption a déjà été nommé; l'espoir de justice. L'injuste ne doit pas avoir le dernier mot, le malin doit être puni et le juste récompensé. Il appartient au héros de rétablir la justice là où elle est menacée. Derrière cet espoir se dessine un problème fondamental de toute religion, le problème de la théodicée: l'espoir se tourne vers une ultime justice, vers un ordre ou une puissance qui interdisent que l'innocent souffre.

Selon une quatrième analogie structurelle on trouvera, et dans le romanfeuilleton et dans la religion, le personnage porteur et garant du salut, le personnage charismatique qui rend possible ce salut. Bien sûr, il est soumis aux souffrances, mais il remporte la victoire face à la puissance du mal. Son oeuvre salutaire est consacrée aux autres, il les libère, il les soutient, il les console.

La cinquième analogie apparaît du côté du lecteur dans sa perception du roman. Normalement, nous l'avons dit, le lecteur est entraîné dans un processus dramatique qui le fait traverser des angoisses et des misères pour lui faire vivre le bonheur. En parlant d'Aristote et du drame antique, nous avons déjà essayé de déceler, au niveau du vécu, les analogies par rapport à une institution religieuse: celle du théâtre de l'antiquité. Or, des structures semblables se montrent au niveau de la liturgie chrétienne qui souvent organise la dramaturgie de son rite comme un cheminement qui mène de la confession des péchés à la promesse ou à l'appropriation sacrementelle du salut. De son côté, à l'issue d'un chemin d'angoisse (par compassion), le roman accordera à son lecteur un sentiment de grand soulagement : son espoir de rédemption est réalisé. Sous un aspect purement fonctionnel on peut donc parler ici d'une compensation de frustrations: voilà une fonction analogue à l'une des fonctions de la religion. En plus, nous sommes frappés de constater une analogie par rapport à la religion traditionnelle non seulement sur un plan fonctionnel mais aussi sur un plan substantiel. De part et d'autre il existe, en effet, une équivalence des symboles qui touchent aux expériences fondamentales traitées dans les religions: expérience d'une puissance supérieure qui détermine la vie, expérience d'ultime bonheur et d'ultime souffrance, expérience d'un secours qui - bien plus que secours dans une situation isolée et contingente - est expression d'une expérience fondamentale de la vie humaine. Aussi banal qu'il puisse paraître, le symbolisme des romans se réfère à une réalité ultime, vécue ou espérée.

Or, à la suite de ces analogies, des différences de structure entre le monde des romans et la religion viennent s'imposer. 
Une première différence est marquée par le fait que la réalité narrée des romans ne réclame aucune autorité interprétative. Bien sûr, ni le drame antique ni même les mythes des religions ne prétendent avoir une telle autorité. Toutefois, le drame et la mythologie se trouvent intégrés dans tout un système global d'orientations, système socialement reconnu, système d'interprétation du monde, de la société, de l'existence individuelle. Un tel horizon d'interprétation généralement accepté manque aux romans-feuilletons - à moins qu'on le perçoive dans les schèmes fondamentaux d'interprétations fournis dans nos sociétés actuelles.

Au niveau même où les religions expriment la revendication de leur autorité - niveau marqué aujourd'hui par une pluralité d'interprétations possibles -, les interprétations implicites des histoires de romans n'arrivent pas à s'imposer d'autant plus que ni auteurs ni lecteurs n'affirment des revendications de cet ordre. Or, les systèmes élaborés des religions d'aujourd'hui prétendent toujours disposer d'une telle autorité d'interprétation. Le monde du roman fait allusion à cette autorité sans la rendre explicite et, par conséquent, sans déclencher le potentiel d'une réflexion religieuse.

Si l'on part - pour parler d'une seconde différence de structure - de l'idée de Luhmann selon laquelle la religion sous un aspect fonctionnel contribue à rendre "l'indéterminable» du monde plus «déterminable», il faut admettre que le monde fictif des romans est loin d'accomplir une telle fonction. Au mieux, il y arrive dans les cas où les romans correspondent au désir d'un monde familier et transparent: la simple dichotomie entre le bien et le mal - bien identifiables - paraît comme une tentative de rendre déterminable (ou apparemment déterminable) l'indéterminable. Mais que signifie sous cet aspect le fait que les personnages symboliques soient interchangeables? Si dans le Western le malin c'est "Concho», ce sera le «Docteur Marius» dans le roman de médecin: le mal n'est donc manifestement pas condensé dans un concept universel ni interprété comme une contre-puissance universelle. Puisque l'expérience du mal reste indéterminée, sa symbolisation reste aléatoire.

On arrive à des conclusions analogues en s'interrogeant sur la force interprétative du concept de «destin» par opposition au concept de Dieu. Le Dieu de la tradition judéo-chrétienne est un dieu personnel : Dieu est conçu comme un dieu qui se tourne vers son peuple, comme une personne qui décide et qui agit. L'indéterminable d'une puissance supérieure est, en effet, rendu déterminable par une personne malgré un part d'indéterminable qui persiste (Deus absconditus). Même dans une version "déiste», le concept de Dieu reste toujours basé sur l'idée d'un ordre global de l'univers: l'ordre y règne, le chaos est surmonté, l'indéterminable est donc rendu déterminable. Or, l'idée du «destin » telle qu'elle apparaît dans les romans détermine peu de choses d'une puissance supérieure: elle l'exprime, c'est tout. Le happy-end de bonheur et de salut qui marque régulièrement la fin des romans satisfait le lecteur qui, lui, le dévoile en même temps comme irréel.

Troisième différence: sous une interprétation religieuse, la rédemption est conçue comme universelle, fondée sur une volonté divine universelle, ancrée dans un principe structurel de valeur cosmique. La rédemption individuelle est une participation à cette action rédemptrice conçue comme universelle dans un sens qui va jusqu'aux profondeurs de l'existence. Dans les romans, la rédemption est symbolisée par la solution des problèmes de la vie. 
Il est vrai que ces problèmes sont présentés comme absolument déterminants pour toute l'existence - ce qui confirme le caractère symbolique et mythique de certains romans -, mais c'est toujours le problème individuel qui reste au centre de l'intérêt: il ne s'agit jamais que de solutions partielles. Là encore, la contingence n'est pas supprimée, elle est précisément maintenue.

Dans les religions, la rédemption constitue un événement qui dépasse le monde actuel de notre expérience et qui implique donc une dimension de transcendance, symbolisée par l'idée d'une transcendance spatiale (ciel) ou temporelle (avenir, Royaume de Dieu, union originelle). La plupart des romans, par contre, restent dans une perspective purement immanente: ce sont les problèmes de la vie quotidienne qui sont à résoudre, le bonheur et la justice à l'heure actuelle étant le sujet principal - peut-être parce qu'une transcendance ne semble guère pensable. Si toutefois une transcendance semble se dessiner plus clairement - comme en science-fiction, par exemple, elle sera nécessairement et consciemment présentée dans un cadre irréel qui permettra des prises de distance; encore faut-il (toujours en science-fiction) qu'elle soit compatible avec une certaine vue du monde, scientifique et technologique.

Une dernière différence se situe au niveau de l'expérience vécue. Le vécu religieux est enraciné dans la vie sociale ou bien il entraîne la formation de groupements religieux, tandis que le vécu des romans se produit dans la lecture de l'individu qui «suit» cette action racontée, laquelle provoque en lui des projections et des identifications individuelles: peur et satisfaction au moment de la libération. Une fois lu, le roman sera «usé». Un échange communicatif sur le vécu n'aura pas lieu.

En résumé, nos deux tours d'horizon nous présentent un tableau bien ambigu. Toute une série d'analogies nous montre la proximité des mondes du roman et de la religion. D'autre part nous constatons des différences très nettes. La religion se présente comme non-religion et la non-religion comme religion. Ou bien s'agirait-il là d'une nouvelle forme de symbolique et d'expérience religieuses, d'une sorte de religion triviale?

\section{LA «RELIGION TRIVIALE» EN TANT QUE MODE DE PERCEPTION DU MONDE ET DU SOI}

Nous utilisons ici le terme de «religion triviale» de manière tout à fait provisoire pour désigner la dimension religieuse (religieuse au moins de façon analogique) des romans. Ainsi, la question reste ouverte de savoir si on peut parler ici légitimement de religion. Ce n'est qu'après un bilan de nos résultats que nous essayerons de donner une réponse à cette question.

Le roman-feuilleton, rappelons-le, fournit une lecture à des couches sociales de scolarité inférieure, souvent à des ouvriers (ouvrières surtout) non qualifiés. D'autre part, il s'agit (chose qui ne va pas de soi) d'un public qui aime lire. Notons encore que ce sont surtout les adolescents et les jeunes adultes jusqu'à l'âge de 30 ans qui s'intéressent à cette littérature, une tranche d'âge qui souvent a pris ses distances à l'égard de la religion institutionnelle, à savoir face aux Eglises en RFA. Il se peut que cette prise de distance s'explique aussi par le fait que les jeunes gens de scolarité limitée préfèrent traiter 
des problèmes de leur vie sur un plan de vécu plutôt que sur un plan doctrinal alors que ce dernier paraît être dominant dans les Eglises, et dans l'Eglise protestante en particulier.

Nous avons pu constater que la religion institutionnelle et chrétienne tient une place bien précise dans les romans-feuilletons. Toutefois, en résumant nos résultats, on peut sans doute dire que la religion institutionnelle de l'Eglise joue, dans les romans, un rôle (si rôle il y a) généralement bien marginal au niveau du vécu. Au moment où les problèmes s'accentuent et où l'on s'approche de la solution, il n'est généralement plus question d'Eglise. La prière (instantanée) que l'on rencontre dans certaines de ces situations reste superficielle et peu déterminante. Est-ce que là encore nous retrouvons le résultat du mouvement de rationalisation qui a marqué le développement de la religion moderne et surtout celui du protestantisme (cfr Habermas 1981, 162ss)? La religiosité façonnée et favorisée par les grandes Eglises, notamment celle de leurs représentants professionnels, est une religiosité rationnelle, réflexive et distante du vécu. La doctrine y a remplacé l'expérience religieuse (cfr Döbert, 106ss). Ainsi se produit un manque de vécu et d'émotionnel en même temps qu'un manque d'expérience de cette réalité qui exige une interprétation religieuse. Dès lors, on ne tient plus compte des besoins religieux de populations entières. Celles-ci se mettent donc à la recherche de nouvelles formes - parfois régressives - d'expériences pour interpréter leur existence, étant donné que le rationalisme des grandes Eglises demande une réflexion discursive d'un accès de plus en plus difficile. Le faible rôle de la religion institutionnelle dans les romans-feuilletons reflète donc une acceptation formelle de l'institution qui fait bon ménage avec une prise de distance et un besoin de résoudre les problèmes religieux de la vie ailleurs que dans cette institution: l'évolution de la religion fait ses victimes. (Peut-être est-ce ainsi que l'on peut caractériser la simultanéité de différents stades du développement de la religion: en ce qui concerne cette simultanéité du non-simultané, voir Bellah, 273).

De tels besoins insatisfaits peuvent aussi favoriser le processus de différenciation religieuse que l'on constate au travers de l'exemple de la «religion triviale». Celle-ci n'est pas simplement un équivalent fonctionnel qui se substitue à la religion : c'est - au moins partiellement - un équivalent fonctionnel proprement religieux. Dans son langage elle aborde le thème religieux même de façon substantielle, le terme du «destin» étant bien un terme religieux. Si ce terme peut avoir des connotations chrétiennes, il exprime, en effet, tout autrement que la tradition chrétienne l'existence d'une puissance supérieure expérimentée dans la vie.

Le mouvement vers une différenciation religieuse que l'on observe dans les romans, le processus de différenciation de la symbolique religieuse, la fin, si l'on peut dire, du monopole symbolique des Eglises chrétiennes dans la tradition occidentale - voilà les conséquences tardives d'un développement qui - depuis l'Aufklärung au moins - atteint la conscience publique : reservé initialement aux élites de la société, ce développement devient progressivement un phénomène de masse. Ce développement fait surgir - ou renaître dans leur indépendance - des institutions qui dans la religion pré-chrétienne se trouvaient solidement intégrées dans un contexte interprétatif religieux: le théâtre, le sport, les beaux-arts et - pour donner un exemple au niveau littéraire - les contes de fées. Dans le cadre de ce mouvement de différenciation 
on trouvera aussi des symbolismes nouveaux qui reprennent également d'anciennes traditions religieuses. Rappelons par exemple la renaissance des symboles de la nature qui ont fait redécouvrir le mer et surtout la montagne, les Alpes. Dans un de ses essais, Georg Simmel a étudié la fascination que peuvent exercer les Alpes. Pour lui, l'expérience de la montagne n'atteint son paroxysme que dans les régions des neiges et des glaces : c'est là qu'elle rejoint «quelque chose de métaphysiquement nouveau, une altitude absolue sans la profondeur qui en est le complément... Ici, le sentiment de libération que nous accordent les moments les plus solennels des paysages des neiges est basé radicalement sur le sentiment de leur anti-position face à la vie " (Simmel, 130). Ce que dit Simmel dans un langage philosophique s'exprimera sous forme de roman chez Rudolf Stratz. Cette fascination devant la nature prête enfin l'arrière-fond émotionnel à un grand nombre de séries de romans de montagnes et de «de pays». Simmel en a trouvé la formule classique: vivre la nature du monde alpin, c'est vivre un fond fascinant de l'être (Seinsgrund). Voilà un langage religieux détaché du christianisme et à la fois l'expression d'un développement plus global de cette différenciation religieuse qui se reflète jusque dans les romans-feuilletons d'aujourd'hui.

D'un autre point de vue encore, la religion des romans-feuilletons pourrait être considérée comme expression d'une interprétation moderne de l'existence. A un niveau trivial, elle exprime l'impossibilité d'une image complète de l'univers qui soit en mesure de fournir une interprétation plausible des contingences et de les rendre supportables en construisant, pour ainsi dire, un horizon global et rassurant qui puisse entourer et consoler l'individu. En général, ce sont aujourd'hui les sciences qui nous fournissent les explications et les interprétations de l'univers. Par conséquent, ce serait aux sciences sociales de prendre en charge aussi les problèmes de la vie individuelle et collective. Or, très manifestement elles ne sont pas à la hauteur de la tâche. Selon Habermas «face aux risques de la vie individuelle, une théorie capable d'éliminer - en les interprétant - les facticités de la solitude et de la culpabilité, de la maladie et de la mort n'est même pas pensable; elle peut, au plus, rendre conscientes en tant que telles les contingences inexorablement liées à la condition physique et morale de l'individu; en principe, il faut donc vivre avec elles sans consolation » $(1973$, p.165). Mais qui serait donc vraiment capable de vivre «sans consolation " ? Devant un tel arrière-plan, de nouvelles formes d'interprétation de l'existence plus consolatrices surgissent sans pour autant construire des images de l'univers (des tentatives, il y en a, bien sûr...) Raconter des histoires de destins contingents qui offrent des possibilités d'identification semble être une manière possible de proposer de la consolation : c'est au moins la solution que proposent les romans. La contingence du destin individuel n'y est pas éliminée: il n'est en effet question que de l'individu. Or, les romans manquent de rigueur dans leur démarche car ils organisent la vie narrée selon une structure figée qui conduira toujours à la réalisation ultime du bonheur, solution illusoire de toute évidence et invitation à une émigration "évasive » de la réalité - non dans le sens d'une évasion collective (qu'offrent par exemple les expériences de peur et de consolation collectives dans le football: voir Lindner) mais d'une fuite de l'individu isolé dans la solitude de sa lecture.

Un dernier aspect ne doit pas être passé sous silence: le monde narré des romans est aussi un produit soumis aux lois du marché. La «religion triviale» est une religion commercialisée qui doit s'accorder strictement à des besoins 
et demandes qu'elle ne saurait modifier qu'avec beaucoup de précaution: elle est obligée de se conformer, d'assumer, de réaliser des attentes sans avoir le droit de critiquer ni la société ni l'individu. A ce point de vue, elle participe tout simplement aux conditions qui semblent déterminer de plus en plus les autres formes de la communication religieuse également. Ainsi, aux USA, les émissions de l'«electronic church" présentent un christianisme bien adapté à la clientèle et au marché. La commercialisation des média en RFA pourrait entraîner des effets semblables. Une religion adaptée au client serait pourtant tout à fait contraire aux intentions des fondateurs des religions classiques. Ces fondateurs sont toujours des personnages prophétiques qui se tournent vers l'avenir pour la changer, qui critiquent, qui enseignent. La question se pose donc à nouveau : cette "religion triviale » est-ce vraiment une religion?

Ingo Mörth nous met en garde devant un concept de religion trop réduit au fonctionnel. Il ne parle de religion que dans les cas où il constate une relation à l'ensemble des inconsistances de l'existence sociale humaine. Pour parler de "religion», il demande en plus la présence de tous les éléments transpersonnels- qui sont constitutifs de la religion. Devant la nécessité de rendre plus flexible un concept aussi rigoureux, il préfère adopter le terme de "phénomènes religieux" comme dénominateur commun pour englober des réalités qui ne relèvent pas de la religion au sens strict. Pour parler des phénomènes qui sortent du domaine délimité de la religion, Mörth utilise des termes comme "équivalents partiels religieux», "protoreligion» ou "religiosité personnelle" (126s). Sans discuter en détail ces termes, on constate que l'effort pour obtenir une définition du concept de religion bien délimité se heurte à la complexité des phénomènes: un élargissement sera nécessaire en regard des cas limites.

Döbert, soucieux lui aussi d'un concept bien précis de la religion, refuse une définition purement fonctionnelle afin de mieux mettre en relief les contenus religieux substantiels des interprétations modernes de l'existence (109ss). Il nous indique une problématique particulière du concept de religion: «le mot'religieux' est tellement associé à la religion organisée que la religiosité personnelle n'y est plus comprise » (115). L'insignifiance relative de la religion qui se manifeste dans les recherches empiriques serait, selon Döbert, au moins partiellement, le résultat artificiel «d'une réduction du terme courant de religion au ritualisme organisé des Eglises» (114).

Au fond, le concept très limité de Mörth ne nous permettrait pas de parler d'une «religion triviale»: les phénomènes décrits ne remplissent pas les conditions de la religion ainsi définie. Mais même dans une telle terminologie pourrait-on parler au moins d'équivalents religieux partiels. Le terme sera pourtant insuffisant face aux phénomènes si on le comprend uniquement dans un sens fonctionnel, car la "religion triviale» comprend aussi des formes substantiellement religieuses, à savoir des interprétations de contingences, des affirmations de puissances supérieures qui déterminent la vie. D'autre part, le terme de religion trop chargé, selon Döbert, par le langage quotidien, semble inutilisable parce que trop lié à l'Eglise institutionnelle (dans le langage allemand, au moins). En tenant compte de ce langage courant au niveau du concept, on arrive à la conclusion suivante: on ne peut pas qualifier de religieux toutes les expressions qui symbolisent la puissance supérieure expérimentée et qui veulent faire face aux contingences, même si - sur un plan 
sur un plan substantiel et fonctionnel - elles équivalent à la religion traditionnelle, car selon leur propre intelligence ces interprétations ne s'entendent pas comme religieuses et ne coïncident pas avec ce que l'on appelle religion dans le langage courant. Bref, la difficulté vient du fait qu'il existe une religion qui se définit comme non-religion - et peut-être il existe même une non-religion qui prétend être religion. Le terme de religion n'est donc plus adéquat pour désigner tous les phénomènes qu'il devrait comprendre. D'une part, il ignore des phénomènes religieux qui sont vraiment religieux, d'autre part, il déclare religieux des phénomènes qui - bien que situés dans le contexte de la religion institutionnelle - ne relèvent pas de la religion au sens strict du terme. Aussi, pour parler de tous les phénomènes qui ont pour fonction l'interprétation du monde et du soi, il convient d'utiliser une catégorie plus formelle et historiquement moins chargée. L'interprétation du monde et du soi peut alors se faire plus généralement dans l'acceptation d'une puissance supérieure qui détermine la vie. La religion ne serait donc qu'une certaine forme historique d'une telle interprétation. Et qu'en est-il de la «religion triviale»? Là encore il s'agirait d'un mode d'interprétation du monde et du soi - presque un mode d'expérimenter le monde et soi-même: expérience fictive bien sûr selon la dramaturgie de la narration, mais expérience tout de même. Redisons-le, la difficulté du langage et de la classification conceptuelle réside dans les faits mêmes, à savoir dans un processus de différenciation de la religion dont les limites deviennent vagues. La religion est la - elle est nulle part et partout à la fois. Mais le problème qui fait sa raison d'être persiste : face à l'expérience d'une puissance supérieure à la vie, les hommes se trouvent devant la nécessité de trouver une asurance - dans le rituel ou dans les mythes, dans la réflexion ou dans le vécu, dans des structures bien élaborées ou dans des structures très simples, dans l'esthétique, dans la philosophie ou bien dans la trivialité.

\section{Bibliographie}

ALBRECHT, H., Arbeiter und Symbol, Kaiser/Grünewald, München, 1982.

ARTAUD, A., Das Theater und sein Double, Fischer, Frankfurt a.M., 1979;

BELLAH, R.N., Religiöse Evolution. In: Seyfarth, C. und Sprondel, W.M. (Hg.), Seminar : Religion und gesellschaftliche Entwicklung, Suhrkamp, Frankfurt a.M., 1973.

BURGER, C., Textanalyse als Ideologiekritik, Athenäum, Frankfurt a.M., 1973.

DOBERT, R., Religiöse Erfahrung und Religionsbegriff, In: Religionspädagogische Beiträge 14/1984, 98-118.

ERMERT, K. (Hg.), Neugier oder Flucht? Zur Poetik, Ideologie und Wirkung der ScienceFiction, Klett, Stuttgart 1980.

GIESEN, R., Der fantastische Film Teil 1 und Teil 2. Roloff und Seeßlen, Schondorf/Ammersee, 1980.

GROEBEN, N., Mythos contra Erklärung: Dimensionen eines psychologischen Konflikts. In : Wermke, J., Comics und Religion, 137-167.

HABERMAS, J., Legitimationsprobleme im Spätkapitalismus. Suhrkamp, Frankfurt a.M., 1973.

HABERMAS, J., Theorie des kommunikativen Handelns Bd, I. Suhrkamp, Frankfurt a.M., 1981.

JABS-KRIEGSMANN, M., Zerrspiegel: Der Deutsche Illustriertenroman 1950-1977. KlettCotta, Stuttgart, 1981. 
KAES, A.; ZIMMERMANN, B. (Hg.), Literatur für viele 1. Zietschrift für Literaturwissenschaft und Linguistik, Beiheft 1, Vandenhoeck \& Ruprecht, Göttingen, 1975.

KILLY, W., Deutscher Kitsch, Vandenhoeck \& Ruprecht, Göttingen, 1961.

KRAUSS, H., Religiöse Themen und Kategorien in der Science Fiction. Parallelen und Abgrenzungen. In: Ermert, Neugier oder Flucht? 95-105.

KREUZER, H. (Hg.), Literatur für viele 2. Zeitschrift für Literaturwissenschaft und Linguistik, Beiheft 2, Vandenhoeck \& Ruprecht, Göttingen, 1976.

LINDNER, R. (Hg.), Der Fußballfan, Syndikat, Frankfurt a.M., 1980.

LIPP, W., Institutionen - Mimesis oder Drama ? In: Zeitschrift für Soziologie 5/1976, 360-381.

LOWENTHAL, L., Literatur und Massenkultur, Schriften Bd. I, Suhrkamp, Frankfurt a.M., 1980.

LUHMANN, N., Die Organisierbarkeit von Religionen und Kirchen, In : Wössner, J. (Hg.), Religion im Umbruch, Enke, Stuttgart, 1972.

MALETZKE, G., Massenkommunikation, In : Handbuch der Psychologie, 7. Bd., Sozialpsychologie, Verlag für Psychologie, Göttingen, 1972.

MALLINCKRODT, A.M., Das kleine Massenmedium. Verlag Wissenschaft und Politik, Köln, 1984.

METZ, W., Kirche und Religion in den Illustrierten, Calwer-Verlag, Stuttgart, 1971.

MORTH, I., Die gesellschaftliche Wirklichkeit von Religion, Kohlhammer, Stuttgart/Ber$\operatorname{lin} / \mathrm{Köln} /$ Mainz, 1978.

NOELLE-NEUMANN, E. (Hg.), Allensbacher Jahrbuch der Demoskopie 1976-1977, Molden, Wien/Zürich/Innsbruck, 1976.

NUSSER, P., Romane für die Unterschicht, J.B. Metzler, Stuttgart, ${ }^{5} 1981$.

NUSSER, P., Zur Rezeption von Heftromanen, In: Rucktäschel/Zimmermann, Trivialliteratur, 61-79.

OHLIG, K.-H., Comics und religiöse Mythen, Oder : Was Religion und Unterhaltungsliteratur nicht gemeinsam haben, In: Wermke, J., Comics und Religion, 121-136.

PFORTE, D., Bedingungen und Formen der materiellen und immateriellen Produktion von Heftromanen, In: Rucktäschel/Zimmermann, Trivialliteratur, 30-60.

PLAKE, K., Die Sozialisationsorganisationen, Westdeutscher Verlag, Opladen, 1981.

RUCKTASCHEL, A.; Zimmermann, H.D. (Hg.), Trivialliteratur, Fink, München, 1976.

SCHAFER, T., Artikel Traktatgesellschaften, In: Real-Enzyklopädie für protestantische Theologie und Kirche, 15. Bd. J.C. Hinrichs'sche Buchhandlung, Leipzig, 1985, 791-803.

SCHENDA, R., Volk ohne Buch. Deutscher Taschenbuchverlag, München, 1977.

SCHENK, M., Publikums - und Wirkungsforschung, Mohr, Tübingen, 1978.

SCHMIDTCHEN, G., Lesekultur in Deutschland 1974, In: Archiv für Soziologie und Wirtschaftfragen des Buchhandels XXX, Börsenblatt für den deutschen Buchhandel - Frankfurter Ausgabe - Nr. 39, 17. Mai 1974.

SIMMEL, Georg, Philosophische Kultur, Wagenbach, Berlin, 1986.

SPEETZEN, R., Romanhefte - Die vergessenen Medien, In: Interview und Analyse 6/1979, 537-543.

SPEETZEN, R., Neue Ergebnisse zum Roman-Markt, In: Interview und Analyse 8/1981, 307-310.

WERMKE, J. (Hg.), Comics und Religion, Fink, München, 1976. 


\section{LIVRES REÇUS / BOOKS RECEIVED}

Al-Assiouty, Sarwat Anis, Théorie des Sources, Recherches Comparées sur le Christianisme primitif et l'Islâm premier, Letouzey \& Ané, Paris, 1987, p. 82.

ADRIANCE, Madeleine, Opting for the poor, Brazilian Catholicism in Transition, Sheed \& Ward, Kansas City, 1986, $200 \mathrm{p}$.

Follmann, José Ivo, Igreja, ideologia e classes sociais, Vozes, Petrópolis, 1985, 208 p.

Furman, D., Religion and Social Conflicts in the U.S.A., Progress Publishers, Moscow, 1984, p. 251.

Renaerts, Monique, La mort, rites et valeurs dans l'Islam Maghrébin, Le Monde Musulman Contemporain, Initiations, U.L.B., Bruxelles, 1986, p. 126.

Rosado Nunes, Maria José F., Vida religiosa, nos meios populares, Vozes, Petrópolis, 1985, p. 297.

TENTORI, Tullio, L'informazione religiosa nella stampa italiana, Franco Angeli, Milano, 1986, p. 403.

Weyand, Winfried, Schriftenverzeichnis Joseph Höffner 1933-1983, Kremer, Köln, 1986, p. 427. 